\title{
SEXO SEM PRESERVATIVO SOB A ÓTICA DAS REPRESENTAÇÕES SOCIAIS DE ACADÊMICOS DE UMA UNIVERSIDADE PÚBLICA
}

\author{
SEX WITHOUT A CONDOM UNDER THE VIEW OF ACADEMIC SOCIAL \\ REPRESENTATIONS OF A PUBLIC UNIVERSITY
}

\author{
José Andrade Almeida Júnior ${ }^{1}$, Diego Gabriel Santos de Oliveiraa ${ }^{1}$, Cleuma Sueli Santos Suto ${ }^{1}$, \\ Carle Porcino ${ }^{2}$, Laura Emmanuela Lima Costa ${ }^{1}$ \\ Universidade do Estado da Bahia - UNEB ${ }^{1}$, Universidade Federal da Bahia - UFBA ${ }^{2}$
}

\begin{abstract}
The objective is to apprehend the academic social representations of a public university on the nonuse of condoms in sexual relations. It's a descriptive and qualitative study. Conducted with the participation of 215 students from a public university, from March to December 2017, for data gathering. Most of the participants were women, Christians, heterosexuals with an active sex life. It's important to refer that a considerable percentage of women said they did not like using condoms and / or did not have direct access to it. From the evocations, a picture of four houses, bringing a central core with the terms "Sexually Transmitted Infections, Pregnancy, Unwanted Pregnancy and Risks", two adjacent peripheries and a distant one. In the central nucleus, the participants highlight the negative aspects of unprotected sex, pointing out the fear of sexually transmitted infections and an unwanted pregnancy; however it opposes this perspective when assuming a sexual attitude seen as risky.
\end{abstract}

Key words: Sexually Transmitted Diseases; Sexuality; HIV; Condoms; Students.

\section{Resumo}

Apreender as representações sociais de acadêmicos de uma universidade pública sobre o não uso do preservativo nas relações sexuais. Estudo descritivo e qualitativo. Realizado com participação de 215 estudantes de uma universidade pública, no período de Março a Dezembro de 2017, para coleta de dados. Participantes em sua maioria mulheres, cristãs, heterossexuais com vida sexual ativa. Chamando atenção que parcela considerável afirmou não gostar de usar o preservativo e/ou não possuir acesso direto. Das evocações, um quadro de quatro casas, trazendo um núcleo central com os termos "Infecções Sexualmente Transmissiveis, Gravidez, Gravidez indesejada e Risco", duas periferias próximas e uma periferia distante. No núcleo central, os participantes evidenciam os aspectos negativos do sexo sem proteção, apontando o medo de infecções sexualmente transmissíveis e uma gravidez indesejada, no entanto contrapõe essa perspectiva ao assumirem uma postura sexual vista como arriscada.

Palavras-chave: Doenças Sexualmente Transmissíveis; Sexualidade; HIV; Preservativos; Estudantes. 


\section{Introdução}

O uso do preservativo, é atualmente, o método de maior utilização e apontado como mais eficaz na prevenção das infecções sexualmente transmissíveis (IST), bem como na prevenção de gravidez não planejada. Apesar disso, sabe-se que as taxas de uso costumam ser frequentes apenas nas primeiras relações sexuais. Esse processo pode ser explicado devido ao fato das relações tornarem-se mais intimas com o tempo e gerar nos indivíduos uma sensação de segurança em sua parceria ${ }^{1}$.

É realidade no Brasil e no mundo as dificuldades para o uso contínuo do preservativo e que essa situação necessita estar ancorada na ampliação da oferta de novas ferramentas de prevenção ao Vírus da Imunodeficiência Humana (HIV). As novas tecnologias possuem limitações, cada uma dentro daquilo que se propõe, entretanto, são importantes aliadas no combate as infecções pelo HIV e outras infecções sexualmente transmissíveis, quando usadas principalmente aliadas ao preservativo ${ }^{2}$.

Essas tecnologias são necessárias na luta contra as IST, principalmente o HIV, não se tratando de eliminar o preservativo, passando a encara-lo como desnecessário, mas o início da busca pela combinação de estratégias de prevenção, bem como a ampliação das alternativas e ofertas em unidades de saúde ${ }^{3}$.

As regiões Sudeste e Sul apresentam sempre maiores taxas de concentração de IST, no entanto o Norte e Nordeste tem apresentado um maior crescimento linear na taxa de detecção da Síndrome da Imunodeficiência Humana (AIDS), chegando a um aumento de até $66,4 \%$ para o Norte e $35,7 \%$ para o Nordeste em 2016. A faixa etária de maior concentração para os casos de AIDS no Brasil fica com a população entre 25 e 39 anos; os homens representam a maioria dos infectados, mesmo levando em consideração o fenômeno da feminização do HIV/AIDS ${ }^{4,5,6}$.

Estudo sobre Representações Sociais (RS) de pessoas que convivem com o HIV, demonstram o quanto o aporte dessa teoria se faz importante para a compreensão da temática, uma vez que revela a complexidade da construção histórico-social da epidemia, apontando para além das questões objetivas, invadindo o universo imaginário pessoal e coletivo ${ }^{7}$.

As RS são formas de saber e de conhecer sobre um objeto modelado, de forma diretamente legível e isso é feito através de suportes comportamentais, materiais e linguísticos. São baseadas ainda em expectativas e nas necessidades de cada indivíduo, sem perder de vista a coletividade, envolvido com um dado objeto ou situação $0^{8,9}$.

Para pesquisadores e defensores as Representações Sociais abrem um leque de possiblidades e tornam-se valiosos instrumentos na identificação da realidade social, permitindo a construção de novos conhecimentos e saberes ${ }^{(10)}$ Por se mostrar tão eficaz em pesquisas cientificas, esse estudo utilizou a Teoria das Representações Sociais (TRS), para dar ancoragem aos resultados seguidamente revelados, e os pressupostos de Abric ao ampliarmos o olhar teóricometodológico à Teoria do Núcleo Central ${ }^{11}$.

A pesquisa aqui apresentada justifica-se pela necessidade de suprir demandas e questionamentos acerca de pensamentos, atitudes e práticas sexuais de jovens e adultos, proporcionando a profissionais da área da saúde e estudiosos uma ampliação dos olhares para as tecnologias de prevenção as IST/HIV/AIDS. Podendo ser útil ainda para os demais leitores, ajudando-os a repensar as ações frente ao cuidado com sua saúde sexual e reprodutiva.

Expõe-se para tanto como questionamento: Quais as representações sociais de discentes de uma universidade pública acerca do não uso do preservativo nas relações sexuais e como objetivo: Apreender as representações sociais de acadêmicos de uma universidade pública sobre o não uso do preservativo nas relações sexuais.

\section{Metodologia}

Tratou-se de um estudo descritivo com abordagem do tipo qualitativa. As pesquisas descritivas focam-se em descrever características que sejam inerentes a uma dada população ou mesmo de um fenômeno, causando a demonstração do elo entre objetos que são variáveis ${ }^{12}$. Sobre a abordagem qualitativa, a mesma é concebível quando o pesquisador busca a compreensão da ocorrência de fenômenos e seus possíveis movimentos ${ }^{13}$.

A TRS foi utilizada para dar ancoragem a esse estudo, uma vez que nos leva a compreender que a mesma cria oportunidades de apreender sobre o cuidado numa ótica mais ampla sobre o ser humano e suas formas de ver e 
interagir com o mundo ${ }^{14}$.

A reflexão feita sobre a teoria, é quanto à forma como os sujeitos, individualmente $e$ coletivamente, constroem-se através do contexto ao qual estão inseridos, e de como a cultura e o ambiente os influenciam. Em resumo, busca-se pela interação humana com a sua realidade, as influências e os resultados dessa interação que são pautadas na comunicação ${ }^{15}$.

Colaboraram com esta pesquisa 215 estudantes dos sexos masculino e feminino, de uma universidade pública, com representação de todos os cursos existentes no período da coleta dos dados, licenciaturas: Pedagogia, Matemática e Ciências Biológicas e bacharelados: Ciências Contábeis e Enfermagem. De cada curso participaram duas turmas, onde o critério de escolha foi o semestre da graduação, sendo uma de início de formação (10 ou 2o semestre) e a segunda em processo de finalização do curso (7음 ou 8 o semestre).

Os critérios de inclusão para a participação foram: Ter idade igual ou superior a 18 anos no momento da coleta; ser aluno/a regularmente matriculado/a e cursando um dos cursos de graduação. Para exclusão: Discentes que não estivessem na sala no momento da coleta.

O local utilizado para coletar os dados foram as salas de aula da Universidade do Estado da Bahia - UNEB, campus VII, situada no município de Senhor do Bonfim, no período de Março à Dezembro de 2017.

A associação livre de palavras foi a técnica utilizada para captar as RS dos participantes, através de um instrumento de coleta composto pelo termo indutor "SEXO SEM PRESERVATIVO", onde as evocações foram inscritas pelos próprios participantes em formulário específico. Em seguida, os/as participantes responderam a um questionário de caracterização, onde questões como sexo, idade, condição sexual, religião, aspectos sexuais, dentre outras, foram levantadas.

Para fins de organização e manutenção do anonimato dos participantes, os instrumentos foram codificados com variáveis préestabelecidas, onde cada variável recebeu uma numeração contendo três ou dois dígitos sendo digitados sequencialmente e separados por ponto e vírgula. O software utilizado para construção desse banco de dados (corpus) foi o Microsoft Word 2010.
O corpus passou ainda por um processo de aproximação lexical das palavras ou expressões evocadas, que consistiu em agrupar palavras ou expressões que tivessem aproximação significativa, mesmo que morfologicamente diferentes. Esse processo é de grande importância à pesquisa uma vez que torna os resultados mais consistentes e facilita a análise e discussão.

O processamento final dos dados foi realizado através do software EVOC (Ensemble de Programmes Pemettant L Analyse des Evoctions) Versão 2005, que possibilitou a organização das evocações através da hierarquia implícita, da frequência com que essas palavras foram evocadas e também através da ordem natural das evocações, expondo assim o Rang das palavras ou expressões evocadas, sendo este, portanto, o valor representativo de quão prontamente e primeiramente foram anunciadas. O EVOC trouxe a luz um quadro de quatro casas onde as maiores frequências e as ordens médias são os critérios considerados ${ }^{16,17}$.

O quadro traz consigo a ordenação das evocações dividindo-as em quatro quadrantes, onde o superior esquerdo revela o núcleo central das RS dos participantes, seguido do superior direito que está intimamente ligado ao núcleo central e deve ser observado com cautela. 0 quadrante inferior direito dispõe-se as evocações com menor frequência e que ocupa as últimas posições. O último quadrante corresponde aos elementos de contraste ao núcleo central, caracterizando a formação de um possível subgrupo ${ }^{18}$.

Em respeito aos fins éticos e legais esta pesquisa está em consonância com as diretrizes da resolução 466 de 2012, tendo sido aprovado pelo Comitê de Ética em Pesquisa da UNEB em 02 de Março de 2017 sob número de parecer 1.945.573.

\section{Resultados e Discussão}

A maioria dos discentes da UNEB-Campus VII autodenominaram-se pertencentes a classe feminina, consolidando $74,4 \%$ do total de entrevistados, a faixa etária que prevaleceu foi na margem entre 20 e 30 anos, onde representaram 67,4\%. A diluição por curso e semestre de pertencimento não demonstrou grandes variações, tendo o curso de pedagogia o maior percentual, concentrando $27,5 \%$ do total 
geral de participantes.

No tocante a Religião/Crença/Denominação, $74,9 \%$ são cristãs(os), subdivididas(os) em maioria católica e evangélica. 92,5\% referiram-se como heterossexuais. Quando questionados (as) sobre sua inserção numa relação de união estável, 68,4\% disseram não se enquadrar nesse quesito, ao passo que $70,7 \%$ declararam já possuir vida sexual ativa. Ainda falando sobre sua vida intima/sexual, 43,2\% disseram gostar de usar o preservativo nas relações sexuais. Sobre o acesso ao preservativo, $40 \%$ afirmaram que o compram em estabelecimentos comerciais, e chama atenção que $20 \%$ disse não ter acesso ao mesmo. Quando questionados (as) sobre o número de parcerias sexuais no período referente aos últimos doze meses (usando por base o dia da coleta de dados) $55,8 \%$ referiram ter tido apenas um(a) parceiro(a).

Figura 1: Quadro de quatro casas das evocações. Senhor do Bonfim, BA, Brasil, 2018

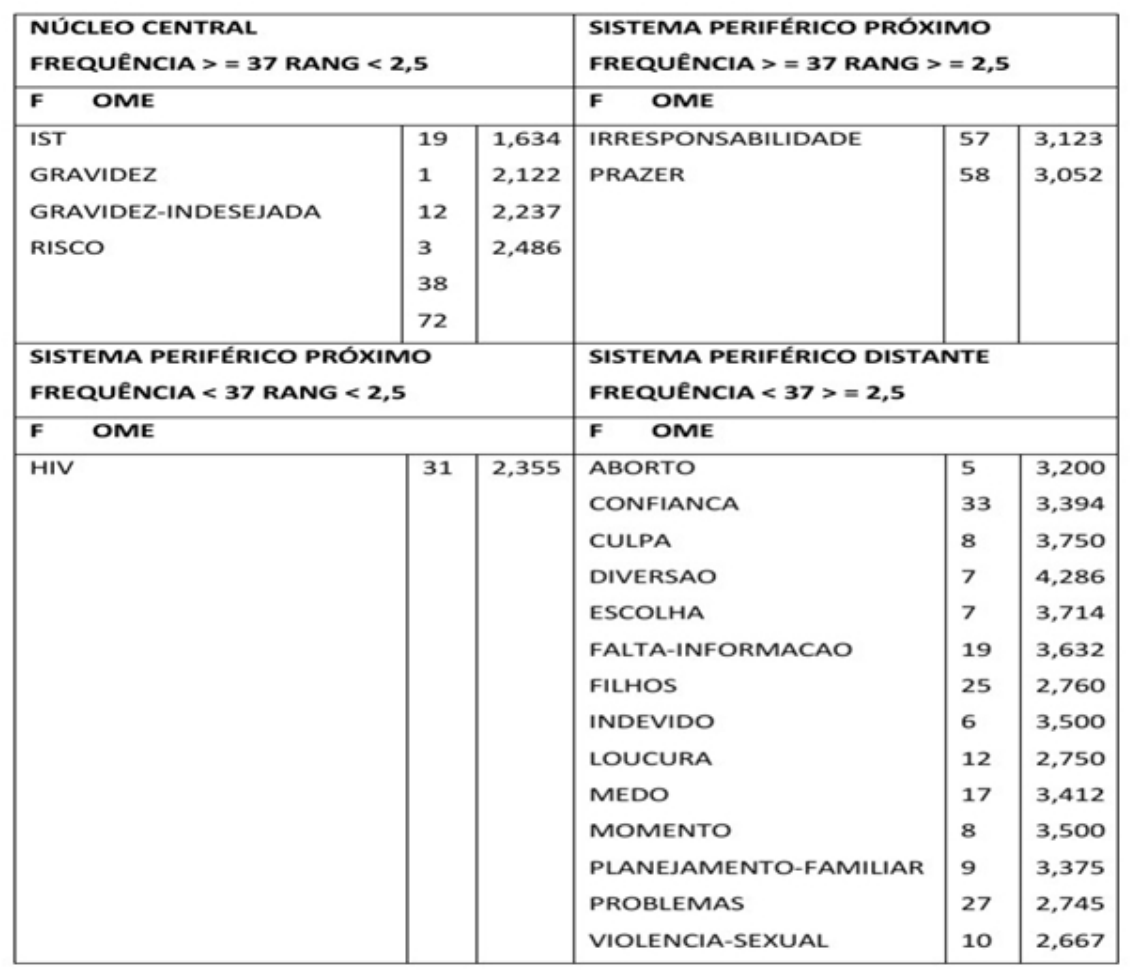

O termo "IST" localizado no quadrante superior esquerdo da Figura 1, compõe o provável núcleo central das representações dos entrevistados e, foi evocado durante a pesquisa 191 vezes (88,9\%). Além de ter sido a palavra com maior frequência, foi ainda a que teve o menor Rang (1,634), isto é, apareceu quase sempre como as primeiras lembranças dos participantes ao se depararem com o termo indutor "sexo sem preservativo". Ainda configurando o núcleo central apareceram os termos Gravidez, Gravidez-Indesejada e Risco.

Infecções sexualmente transmissíveis, são afecções de saúde, que tem como agente etiológico, variados microrganismos e são adquiridas pelo ser humano através do contato sexual desprotegido, por meio de fluídos oral, vaginal e anal. A transmissão é possível ainda por meio da gestação, parto e amamentação, onde a mãe torna-se a transmissora para o bebê ${ }^{4}$.

IST são geradoras de representações sociais nos meios de relação humana e alavancam verdadeiras discussões políticas por todo o mundo na busca do tratamento, cura, prevenção ou mesmo para o combate. A força representativa do termo IST, aponta o quanto o discurso normativo das campanhas de prevenção da AIDS e outras infecções, desperta nas pessoas o imaginário do impacto de se descobrir infectado, sem, contudo, pensar naqueles que já convivem com o HIV e as repercussões que esse tipo de campanhas intimatórias pode trazer ${ }^{19}$.

É preciso ter cautela ao se trabalhar campanhas de prevenção, para não reproduzir 
através de mídias os estigmas criados pela sociedade civil ou mesmo as sociedades médicas, como o que foi feito com o início da epidemia da AIDS nas décadas de 80 e 90, uma vez que tão importante quanto a prevenção, é o cuidado com as pessoas já infectadas, propiciando a essas, uma base sólida e segura para a aceitação pós diagnóstico, início e continuidade do tratamento ${ }^{19}$.

As infecções sexualmente transmissíveis elevam os custos e gastos com tratamentos e por isso a prevenção ainda é a alternativa mais viável, entretanto, são necessários esforços contínuos, uma vez que, se as campanhas ou programas não levarem em consideração as questões sociais dos públicos que se deseja alcançar, o nível de conhecimento não irá se elevar, tampouco as atitudes e práticas irão sofrer mudanças ${ }^{20}$.

Ao se falar em prevenção, o ideário a ser sustentado é o de pensar em alcançar as perspectivas dos jovens, abandonando filosofias baseadas na verticalização das ações, buscando assim novas ferramentas e propostas no combate as IST/HIV/AIDS ${ }^{21}$.

Quando se fala em sexo sem preservativo, em primeiro momento é nítida a reprovação social, principalmente nos meios com níveis escolares ou acadêmicos mais elevados ${ }^{1}$. Com os participantes desta pesquisa esse fluxo foi seguido sem desvios de padrão, isso se percebe com clareza ao se deparar com o núcleo central acima, onde a ideia de negativismo se coloca sem recuos, até mesmo em se tratando de uma gravidez.

O que chama atenção é que mesmo trazendo à tona as repercussões negativas do sexo desprotegido, 34,9\% dos entrevistados afirmaram não gostar de usar o preservativo nas relações sexuais, devendo ainda levar em conta que praticamente $22 \%$ optou por não responder a essa questão. Isso nos leva a buscar respostas do porquê desse dualismo e mais ainda, qual a motivação que muitas pessoas utilizam para praticar sexo sem a proteção de um método de barreira, que é considerado o mais eficaz na prevenção do HIV/AIDS e da gravidez nãoplanejada, que é o caso dos preservativos masculino e feminino ${ }^{22}$.

O uso do preservativo tem relação com muitas variáveis. Uma dessas é o nível econômico da população, onde classes com maior poder aquisitivo costumam apresentar baixo percentual de relações sexuais protegidas pelo preservativo.
Esse fato pode estar intimamente ligado com a maior facilidade de aquisição de outros métodos contraceptivos que arriscadamente não oferecem dupla proteção ${ }^{23}$. Outra relação interessante com a aceitação do preservativo é o grau de instrução escolar, onde, essa variável, não se faz suficiente para garantir a regularidade do sexo protegido 24 .

Gravidez e Gravidez-indesejada, vieram logo após IST e são seguidas pelo pensamento de risco. O núcleo central desse estudo, se mostra muito sólido em relação ao que os participantes representam sobre fazer sexo sem uso do preservativo. Eles seguem exatamente o itinerário dos objetivos da camisinha, evitar a contaminação por infecções e evitar a gravidez de pessoas que não pretendem ter filhos. Portanto, sexo sem proteção gera riscos de contrair doenças e de conceber um filho ${ }^{22}$.

Fato importante para a assunção de riscos nas relações sexuais desprotegidas são as questões de gênero e de confiança em parcerias sexuais. Cerca de $40 \%$ dos entrevistados em uma pesquisa acreditam não ser necessário usar preservativo nas relações sexuais e que ainda cerca de $20 \%$ se sentiriam incomodados(as) ou mesmo insultados(as) caso sua parceria solicitasse o uso, apontando ainda que jovens masculinos estão mais propensos a comportamentos de risco que as jovens e que o fato se deve a baixa percepção de risco por parte desse público ${ }^{20}$.

A questão da confiança nas parcerias, é um fenômeno de ocorrência em pessoas de ambos os sexos masculino e feminino, quando afirmam em seus discursos que a estabilidade da relação pode ser vista como medida preventiva às infecções sexualmente transmissíveis, uma vez que para essas pessoas conhecer a parceria por um determinado período de tempo se faz suficiente para "tomar o lugar do preservativo"21.

No quadrante superior direito da Figura 1, apresenta-se a primeira periferia, é importante dar visibilidade aos termos que aparecem nesse sistema, sendo eles Irresponsabilidade, evocado 57 vezes e Prazer, 58 vezes. Mesmo considerando que os dois termos apresentaram Rangs de 3,123 e 3,052 respectivamente, ou seja, foram evocados mais tardiamente, estes dão sustentação ao núcleo central ao explicar os termos evocados neste mesmo núcleo.

Utilizar o prazer para motivar/justificar o sexo sem preservativo também se mostrou 
presente em outro estudo, apontando que para estudantes de uma rede pública estadual de ensino básico, o sexo sem proteção com método de barreira é tido como algo que traz maior sensação de prazer, quando comparado à relação com preservativo. Assinalando que tal ato é compreendido pelos praticantes como algo incorreto, provavelmente representando para eles a irresponsabilidade e assumindo o risco de ser contaminado ou de gerar uma gestação não planejada ${ }^{25}$.

O termo HIV, coloca-se na segunda periferia próxima, no quadrante inferior esquerdo da Figura 1 e, observa-se uma frequência relativamente baixa de 31 evocações, porém deve-se levar em consideração o Rang de 2,355, mostrando que o grupo social aqui analisado deu corpo a essa sigla quase sempre como uma de suas primeiras lembranças, configurando aqui HIV como uma IST que merece destaque especial ao falar sobre o não uso do preservativo. O HIV surge com um destaque importante demonstrando, de certo modo, o quanto o HIV/AIDS marcou a humanidade ao se disseminar rapidamente por todo o mundo, desde os primórdios da epidemia até os dias atuais.

HIV, sigla utilizada para definir o Vírus da Imunodeficiência Humana é responsável por provocar a AIDS, doença na qual os indivíduos afetados, tem seu sistema imunológico comprometido através da destruição dos linfócitos TCD4+, ficando susceptíveis as infecções tidas como oportunistas, reduzindo assim a qualidade de vida das pessoas portadoras do vírus e quando não tratadas podendo ser levadas a morte ${ }^{22}$.

O elo feito entre a configuração e o estigma criado no início da epidemia da Aids, deu-se através da construção da identidade social de pessoas infectadas pelo HIV, onde homossexuais, prostitutas, promiscuidade sexual, magreza e morte, eram o complexo imagético diretamente remetido a síndrome. Trazendo aos dias atuais, consequências para $o$ enfrentamento da doença ${ }^{19}$.

Em abordagens sobre o HIV/AIDS, baseada na teoria das representações sociais, a doença é vista ainda hoje como afecção perigosa, incurável, que leva os sujeitos a morte e que ainda é carregada de estigmas e preconceitos. Esse contexto mostra a importância das pesquisas em representações sociais sobre IST/HIV/AIDS, uma vez que com o aporte da teoria torna-se possível apreender os conceitos de grupos específicos acerca dessa temática, bem como compreender como se dá o processo de proteção e prevenção a exposição e aquisição de infecções sexualmente transmissíveis ${ }^{26,27}$.

No sistema periférico distante, no quadrante inferior direito da Figura 1 apresentam-se termos evocados menos frequentemente e que foram expressos sempre como as últimas lembranças dos participantes: "confiança, falta de informação, medo, aborto, filhos, culpa, loucura".

Apesar de não serem termos sólidos e homogêneos é preciso ampliar o olhar para perceber que alguns desses termos, a exemplo de confiança e falta de informação, provavelmente já estiveram presentes em outros momentos no núcleo central das representações desse e de outros grupos específicos. Sendo esse fato exposto em outros momentos, onde as parcerias são apontadas como 0 argumento principal das pessoas que praticam sexo sem preservativo e que a intimidade é a ferramenta essencial para se estabelecer esse tipo de relação, sendo portanto o uso do método priorizado quase sempre em coito sexual com parcerias eventuais $^{28}$.

Há ainda, correlação entre sexo sem camisinha e questões relacionadas a escolaridade materna e a educação em saúde, indicando que o grau de instrução de mães de jovens e adolescentes fazem diferença, uma vez que a família é uma fonte de informação e se constitui como principal e geralmente primeira base de formação social e sexual dos seres humanos. Profissionais de saúde também devem estar atentos, na busca da conscientização das famílias e sociedade em geral sobre fatores de risco para aquisição de IST e a prática segura do sexo ${ }^{29}$.

O sentido aqui apresentado, pelas (os) estudantes, através dos elementos cognitivos, coadunam com a distinção principal do grupo: cerca de $30 \%$ não iniciaram a vida sexual, mais de $50 \%$ declaram-se monogâmicos, quase 35\% assumem não gostar de usar o preservativo e $20 \%$ declaram dificuldade de acesso. Tais características, justificam que na periferia distante do quadro de quatro casas os elementos evocados apresentem como atributos do não uso de preservativo: conviver com o sentimento de culpa e medo e, assumir uma gravidez ou abortala. 


\section{Conclusão}

Falar de sexo sem preservativo carreia de aspectos negativos, situação que se confirmou através dos termos fortemente evocados e direcionados às infecções sexualmente transmissíveis e a gravidez indesejada, evidenciando medos e consequências sobre o sexo desprotegido. O fato importante é que, mesmo tendo consciência dos riscos, os participantes demonstraram um provável direcionamento a comportamentos vulneráveis perante as práticas sexuais, explicadas através do prazer em transar de forma "livre" e "desimpedida", quando do não uso da camisinha.

Observou-se através desse estudo que as (os) acadêmicas (os), configurando-as(os) como um grupo social rico em informações empíricas e cientificas importantes, trouxeram representações acerca do não uso do preservativo nas relações sexuais, mostrando que a sexualidade, o sexo e as formas de fazê-lo ainda são temas que carecem de pesquisas e respostas às muitas questões que perpassam a temática, principalmente entre a população jovem.

Nesse contexto, ampliar os olhares as novas tecnologias de prevenção as IST/HIV/AIDS que estão disponíveis e buscar meios de oferta-los combinadamente ao uso do preservativo, se faz indispensável, uma vez que o sexo sem proteção é uma realidade constante.

Facilitar o acesso das pessoas às variadas tecnologias de prevenção, proteção ou tratamento não deve ser visto como algo utópico, já que muitas delas são disponibilizadas através do Sistema Único de Saúde, de forma gratuita a exemplo da Profilaxia Pré-Exposição (PrEP), Profilaxia Pós-Exposição (PEP), teste-rápido AntiHIV, Sífilis e Hepatite C, entre outras.

\section{Referências}

1. Borges ALV, Fujimori E, Hoga LAK, Contin MV. Práticas contraceptivas entre jovens universitários: o uso da anticoncepção de emergência. Cad. Saude Publica. [Internet]. 2010 [acesso em 12 de jan 2018]; 26(4). Disponível em:http://dx.doi.org/10.1590/S0102311X2010000400023.

2. Maksud I, Fernandes NM, Filgueiras SL. Technologies for HIV prevention and care: challenges for health services. Rev Bras
Epidemiol. [Internet]. 2015 [acesso em 09 de jan 2018]; 18(1). Disponível em:

http://dx.doi.org/10.1590/1809-

4503201500050008

3. UNAIDS (BR). Prevenção Combinada do HIV. [Internet] Brasília: UNAIDS [acesso em 17 de maio de 2018]. Disponível em: https://unaids.org.br/prevencao-combinada/

4. Ministério da Saúde (BR). Secretaria de Vigilância em Saúde - Departamento de Vigilância, Prevenção e Controle das Infecções Sexualmente Transmissíveis, do HIV/Aids e das Hepatites Virais. Boletim Epidemiológico. Brasília: Ministério da Saúde; 2018.

5. Ministério da Saúde (BR). Secretaria de Vigilância em Saúde - Boletim Epidemiológico: Sífilis 2017. Brasília: Ministério da Saúde; 2017.

6. Ministério da Saúde (BR). Epidemiológico B. Hepatites virais. Ministério da Saúde. 2017;48:1-65.

7. Gomes AMT, Silva ÉMP, Oliveira DC de. Social representations of AIDS and their quotidian interfaces for people living with HIV. Rev Lat Am Enfermagem. [Internet]. 2011 [acesso em 21 de jan 2018]; 19(3). Disponível em: http://dx.doi.org/10.1590/S0104-

\section{6}

8. Jodelet D. O encontro dos saberes. In: Jesuíno JC, Mendes FMM, Lopes MJ (Org.). As representações sociais nas sociedades em mudança. Petrópolis: Vozes, 2015.

9. Brasileiro FS, Azevedo RLW de, Freire GH de A. Representações sociais e necessidades de informação: um estudo no campo arquivístico da Universidade Federal da Paraíba (UFPB). Inf. \& Soc.:Est. [Internet]. 2014 [acesso em 03 fev 2018]; 24(3).

Disponível em:www.periodicos.ufpb.br/ojs/index.php/ies/ar ticle/view/18586

10. Santos MP dos. A teoria das representações sociais como referencial didáticometodológico de pesquisa no campo das ciências humanas e sociais aplicadas. Emancipação [Internet]. 2013 [acesso em 19 jan 2018]. Disponível em: http://www.revistas2.uepg.br/index.php/emanci pacao/article/view/3122

11. Moscovici S. A Psicanálise, Sua Imagem e Seu Público. Rio de Janeiro: Vozes; 2012.

12. Gil AC. Métodos e Técnicas de Pesquisa Social. 6. ed. São Paulo: Atlas; 2008.

13. Minayo MCS. O Desafio do conhecimento: pesquisa qualitativa em saúde. 3 . 
ed. São Paulo: Hucitec; 2006.

14. Ferreira $M$ de $A$. Teoria das Representações Sociais e Contribuições para as Pesquisas do Cuidado em Saúde e de Enfermagem. Esc. Anna Nery [Internet]. 2016 [acesso em 03 fev 2018]; 20(2). Disponível em: http://dx.doi.org/10.5935/1414-8145.20160028

15. Arruda A. Teoria das representações sociais e teorias de gênero. Cad. Pesqui [Internet]. 2002 [acesso em 05 fev 2018]; 117. Disponível em: $\quad$ http://dx.doi.org/10.1590/S0100$\frac{15742002000300007}{16 .}$

16. Vergès P. Ensemble de programmes permettant l'analyse des evocations: manuel version 2. Aix-en-Provence: LAMES; 1999.

17. Gomes AMT, Oliveira DC de. Estudo da estrutura da representação social da autonomia profissional em enfermagem. Rev. esc. Enferm. USP [Internet]. 2005 [acesso em 22 jan 2018]; 39(2). Disponível em: http://dx.doi.org/10.1590/S008062342005000200004

18. Monteiro $\mathrm{P}$ de V, Pereira MLD. E discurso de lo cotidiano y el sentido común: la teoría de las representaciones sociales. Interface - Comun Saúde, Educ. [Internet]. 2013; [acesso em 03 de abr 2018]. Disponível em: http://dx.doi.org/10.1590/S1414-

\section{$\underline{32832013000300020}$}

19. Zambenedetti G, Both NS. "A via que facilita é a mesma que dificulta". Estigma e atenção em HIV-AIDS na Estrategia de Saude da Família. Fractal, Rev Psicol. [Internet]. 2013; 25(1) [acesso em 08 de mar 2018]. Disponível em: http://dx.doi.org/10.1590/S1984-

02922013000100004

20. Fontes MB, Crivelaro RC, Scartezini AM, Lima DD, Garcia A de A, Fujioka RT. Fatores determinantes de conhecimentos, atitudes e práticas em DST/Aids e hepatites virais, entre jovens de 18 a 29 anos, no Brasil. Cien Saude Colet. [Internet]. 2017;22(4) [acesso em 07 de mar 2018]. Disponível em: http://dx.doi.org/10.1590/1413$\underline{81232017224.12852015}$

21. Almeida SA, Nogueira J de A, Goldfarb MPL, Batista FL, Barrêto AJR, Moreira MASP, et al. Concepção de jovens sobre o hiv/aids e o uso de preservativos nas relações sexuais. Rev. Gaúcha Enferm. [Internet]. 2014 [acesso em 11 fev 2018]; 35(1). Disponível em: http://seer.ufrgs.br/index.php/RevistaGauchade Enfermagem/article/view/37074
22. Ministério da Saúde (BR). Departamento de Vigilância, Prevenção e Controle das IST, do HIV/Aids das Hepatites Virais. [Internet]. Brasília: Ministério da Saúde; 2018 [acesso em 17 mai 2018]. Disponível em: http://www.aids.gov.br/pt-br/publicogeral/prevencao-combinada/preservativo.

23. Nascimento EGC, Cavalcanti MAF, Alchieri JC. Adesão ao uso da camisinha: a realidade comportamental no interior do nordeste do Brasil. Rev. Salud Pública. [Internet]. 2017 [acesso em 26 jan 2018]; 19(1). Disponível em: https://doi.org/10.15446/rsap.v19n1.44544

24. Natividade JC, Camargo BV. Social representations, scientific knowledge and sources of information about AIDS. Paidéia. [Internet]. 2011 [acesso em 05 abr 2018]; 21(49). Disponível em: http://dx.doi.org/10.1590/S0103863X2011000200004

25. Bezerra E de O, Pereira MLD, Chaves $A C P$, Monteiro $P$ de V. Social representations of adolescents on sexual relations and the use of condoms. Rev. Gaúcha Enferm. [Internet]. 2015 [acesso em 06 mar 2018]; 36(1). Disponível em: http://dx.doi.org/10.1590/1983-

\subsubsection{9}

26. Sousa LMS, Silva LS, Palmeira AT. Representações sociais de caminhoneiros de rota curta sobre HIV/AIDS. Psicol Soc. [Internet]. 2014 [acesso em 22 jan 2018]; 26(2). Disponível em: http://dx.doi.org/10.1590/S0102-

\section{1.}

27. Suto CSS. Representações Sociais de profissionais de saúde sobre a aids e práticas de cuidado em HIV/aids.[dissertação]. Salvador (BA): Universidade Federal da Bahia; 2015.

28. Rebello LEFS, Gomes R. Qual é a sua atitude? Narrativas de homens jovens universitários sobre os cuidados preventivos com a AIDS. Saude Soc. [Internet]. 2012 [acesso em 18 jan 2018]; 21(4). Disponível em: http://dx.doi.org/10.1590/S010412902012000400011

29. Cruzeiro ALS, Souza LD de M, Silva RA da, Pinheiro RT, Rocha CLA da, Horta BL. Comportamento sexual de risco: fatores associados ao número de parceiros sexuais e ao uso de preservativo em adolescentes. Cienc. saude colet. [Internet]. 2010 [acesso em 12 jan 2018]; 15(1). Disponível em: http://dx.doi.org/10.1590/S1413$\underline{81232010000700023}$ 
Endereço para Correspondência

Rua Silveira Martins no 2555, Cabula

Salvador/BA

CEP.: 41.150-000

andrade.junior320@gmail.com

Recebido em 12/02/2020

Aprovado em 14/05/2020

Publicado em 04/02/2021 